\title{
El colgajo (2018) de Philippe Lançon: una experiencia clínica de la escritura y de la literatura
}

\section{El colgajo (2018), by Philippe Lançon: a clinical experience of writing and literature}

\author{
Jean-Marie Lassus ${ }^{1}$ \\ Universidad de Nantes. Nantes, Francia \\ jean-marie.lassus@univ-nantes.fr \\ ORCID: 0000-0001-5061-3583
}

Citar como: Lassus, J. M. (2019). El colgajo (2018), de Philippe Lançon: una experiencia clínica de la escritura y de la literatura. Desde el Sur, 11(2), pp. 13-22.

Dejadme dolerme, si lo queréis, mas dejadme despierto de sueño, con todo el universo metido, aunque fuese a las malas, en mi temperatura polvorosa.

César Vallejo, «Las ventanas se han estremecido», Poemas en prosa (1923-1929)

\section{RESUMEN}

El objeto de estudio es el relato autobiográfico Le lambeau (El colgajo), de 2018, del periodista y escritor Philippe Lançon, superviviente del atentado contra el periódico satírico Charlie Hebdo del 7 de enero de 2015. Cuenta su estancia en el hospital durante los largos meses de operaciones y reconstrucción facial, en los que la escritura le ayudó a aferrarse a la vida. El artículo se centrará en el universo construido por el narrador entre el relato de vida y la ficción literaria, dentro del marco de una reflexión sobre la atención médica y el humanismo, la salud y la cultura.

\section{PALABRAS CLAVE}

Relato de vida, trauma, arte, cultura, salud

\footnotetext{
1 Jean-Marie Lassus es profesor investigador en Literatura Latinoamericana en la Facultad de Lenguas y Culturas Extranjeras de la Universidad de Nantes y miembro del Centro de Investigación sobre las Identidades Nacionales y la Interculturalidad (Crini) en esta institución. Sus investigaciones tratan de las escrituras de la historia en literatura y de las representaciones de los imaginarios en América Latina. Ha estudiado también las narraciones autobiográficas y la circulación y recepción de las representaciones literarias. Es doctor en Estudios Ibéricos y latinoamericanos por la Universidad de París III, con la tesis Historia y epopeya en el ciclo de La guerra silenciosa de Manuel Scorza, bajo la dirección del profesor Claude Fell.
} 


\section{ABSTRACT}

The object of this study is the autobiographical work by the journalist and writer Philippe Lançon, entitled "Le lambeau» ("Shreds») (2018). Lançon survived the attack on the satirical newspaper Charlie Hebdo on January 7th 2015. He writes of his time in hospital and the long months of surgeries and facial reconstruction procedures, during which writing helped him cling to life. The article focuses on the world constructed by the narrator somewhere between autobiography and literary fiction, as he reflects upon medical care and humanism, health and culture.

\section{KEYWORDS}

life story, trauma, art, culture, health

El colgajo es una narración autobiográfica del periodista y escritor francés Philippe Lançon, uno de los sobrevivientes del atentado contra el periódico satírico Charlie Hebdo del 7 de enero de 2015 en París, reivindicado por Al Qaeda. El texto cuenta en primera persona la hospitalización y los largos meses de operaciones y reconstrucción facial que sufrió el autor, durante los cuales la escritura y la literatura lo ayudaron a aguantar esta situación, mientras la lectura - y la relectura - se volvieron una actividad fundamental, casi vital. Publicado en 2018, el texto de Philippe Lançon tuvo mucha resonancia en Francia ${ }^{2}$, no solo por la emoción que suscitaron las víctimas, sino también por su estilo sosegado y reflexivo y una narración que en ningún momento pide venganza ni entra en polémicas ideológicas. Una escritura que, según la presentación de la editorial Anagrama que publicó la traducción del texto, «traza una estupenda cartografía emocional del individuo vulnerable de nuestros días» ${ }^{3}$. En el universo construido por el narrador entre el relato de vida y la ficción literaria, «la literatura enseña que la enfermedad o la herida pueden ser un vector para tener acceso al mundo de un modo diferente ${ }^{4} \gg$. El texto de Philippe Lançon suscita múltiples reflexiones sobre la relación entre la atención médica y el humanismo, la salud y la cultura, temas abordados en recientes estudios sobre la cuestión en Francia.

2 Publicado el 12 de abril de 2018 por las ediciones Gallimard, El colgajo fue galardonado por el premio literario Femina y Roger Caillois, con una mención especial para el premio Renaudot. La editorial Anagrama, que se encargó de la edición en español, señala su traducción a varios idiomas: alemán, catalán, holandés, inglés, italiano, japonés, polaco y portugués (https://www.anagrama-ed.es/libro/panorama-de-narrativas/el-colgajo/9788433980410/ PN_1009).

3 Anagrama, 2019.

4 Raspiengeas, 2019. 


\section{Entre ficción y realidad, cicatrices y escritura}

Cuando se publicó el texto de Philippe Lançon, la prensa y la crítica literaria usaron diversos términos para calificarlo: Le Monde des Livres habló de «un diario del duelo» ${ }^{5}$, y el periódico Libération de «un testimonio inaudito, tan fascinante como aterrador $\aleph^{6}$. Y mientras el escritor francés Frédéric Beigbeder lo calificaba de «una obra maestra indiscutible, absoluta», el diario quebequés Le Devoir señalaba que para muchos críticos esta novela era el mejor libro de $2018^{7}$. Sin embargo, en este texto de carácter autobiográfico compiten la ficción y la realidad, con una narración principal que se va ramificando en historias privadas y consideraciones sobre la sociedad, y establecen un diálogo permanente con los grandes textos literarios.

En el hospital, las relecturas - tanto o más que las lecturas- modifican la relación del autor con la realidad: Proust, las Cartas a Milena ${ }^{8}$ de Kafka y La montaña mágica (1924) de Thomas Mann son sus «tres espejos deformantes e informativos»'. El autor observa, por ejemplo, que Proust estuvo enfermo la mayor parte de su vida y que tal vez por eso había visto en todas partes solo falsas apariencias, soledad, actitudes, malentendidos», y concluye: «La enfermedad no es una metáfora; es la vida misma» ${ }^{10}$. Kafka le enseña que si uno no puede escapar del infierno en que está ni eliminar la violencia que se le ha infligido puede, en cambio, aprender a vivir con ella y domesticarla buscando la mayor suavidad posible ${ }^{11}$. Es esta suavidad que el narrador encuentra en la poesía, cuando, al sentirse por primera vez en trance de muerte después de un incidente clínico, se recita a sí mismo los últimos versos de «El viaje» de Baudelaire. «La respiración poética» se vuelve entonces tan vital como la máscara que le han puesto: «respirar y respirar esas palabras era más importante, ya que se había convertido en lo mismo» ${ }^{12}$.

En el hospital se produce una progresiva toma de conciencia de las relaciones entre el acto de escritura y su estado, que lo lleva a un cuestionamiento de su identidad «real»:

Cuando escribía en la cama con tres dedos, luego cinco, luego siete, con la mandíbula agujereada y luego reconstruida, con o sin posibilidad

\footnotetext{
5 Birnbaum, 2018.

6 Anagrama, 2019.

7 Desmeules, 2018.

8 Cartas a Milena (1952) reúne algunas de las cartas de Franz Kafka a Milena Jesenská de 1920 a 1923.

9 Lançon, 2018, p. 376. Las referencias de las citas son las de la edición francesa, con las traducciones realizadas por el autor de este artículo.

10 lbíd., pp. 413-414.

11 lbíd., pp. 384-385.

12 lbíd., p. 341.
} 
de hablar, yo no era el paciente a quien describía; era un hombre que revelaba a este paciente observándolo, y que contaba su historia con una benevolencia y un placer que esperaba compartir. Me convertía en una ficción [...] terminaba cada crónica agotado, sudoroso, tosiendo, lloriqueando. El paciente resucitaba de entre las palabras y seguía adelante ${ }^{13}$.

Al apartarle de la agitación de su vida habitual de periodista en continuo contacto con una actualidad brutal y cambiante, su nueva situación lo obliga a concentrarse más en la escritura, a sentir el precio de cada frase y de cada palabra ${ }^{14}$. Escribir se vuelve entonces un acto cada vez más físico, y no tarda en parecerse al acto quirúrgico: cuando su cirujana le explica que la tentación del médico es ir lo más lejos posible en la reconstrucción facial para acercarse a la cara ideal -aun cuando esto nunca se logra y hay que saber detenerse-, él contesta que lo mismo pasa con la escritura: el autor intenta hacer coincidir el libro que está escribiendo con el que imaginaba, pero los dos nunca se juntan, y llega un momento en que tiene que parar también: «El paciente se queda con su boca retorcida, sus cicatrices, su discapacidad más o menos reducida. El libro está solo con sus imperfecciones, sus habladurías, sus defectos» ${ }^{15}$. El texto y el paciente se vuelven ficticios, son como un espejo el uno para el otro y el narrador llega a afirmar que un paciente que no ha sido debidamente operado o reeducado es comparable con un texto fallido ${ }^{16}$. Esta situación recuerda a Michel Foucault ${ }^{17}$, hijo de padres cirujanos, quien declaró percibirse a sí mismo como un cirujano que había sustituido el bisturí con la pluma, considerando la hoja en blanco como el cuerpo de los demás:

Supongo que hay en mi pluma una vieja herencia de bisturí. [...] He pasado de la eficacia de la curación a la ineficacia de los comentarios; he sustituido la cicatriz sobre el cuerpo por el grafiti sobre el papel; he sustituido lo imborrable de la cicatriz por el signo perfectamente borrable y tachable de la escritura ${ }^{18}$.

13 lbíd., p. 366.

14 «Desde hacía algún tiempo, ya no me sentía adaptado a un oficio agitado, inquieto, que exigía adherirse a un mundo que iba demasiado rápido y era demasiado brutal para mí [...] ahora, cada palabra, cada frase, me hacía sentir su precio. Mi mandíbula destrozada tenía una pinta de metáfora y eso no estaba tan mal». Ibíd, p. 183.

15 lbíd., pp. 221-222.

16 «El texto fallido es un paciente que no ha sido debidamente operado o reeducado, $\mathrm{o}$ al que habría sido mejor dejar morir». Ibíd., p. 496.

17 lbíd., p. 464.

18 Foucault, 2011, citado en Torres Garza, 2019. 


\section{II. "La lección de tinieblas» y el impulso vital hacia las otras artes}

Philippe Lançon confiará haber pensado en la posibilidad de un libro desde el invierno de 2016. Tardó un año en terminar El colgajo, tras probar varias formas de alcanzar la mayor naturalidad posible. Al entrar en el hospital, había empezado a escribir por etapas: primero sobre una pizarra y un cuaderno para comunicarse con los demás, ya que no podía hablar; luego mediante los correos electrónicos destinados a su familia y amigos, y por fin redactando sus crónicas periodísticas en Charlie Hebdo. Pero desde los primeros momentos se le planteó la cuestión de la forma, la preocupación por no «sobreexponer» su sufrimiento a sus lectores:

Para salir del grito laberíntico que solo resonaba en mis propios oídos y en mi corazón, tuve que encontrar una forma íntima y clara. A la violencia de la situación, opuse una forma de dandismo. Al escribir en Charlie desde mi cama de hospital, me obligaba a atenerme a mi experiencia de resucitado y de paciente. A narrarla tratando de hacer sonreír, para no aislarme de esta dimensión, nunca ausente, de la vida real ${ }^{19}$.

$\mathrm{Si}$, en su esfuerzo para recordar, el narrador afirma desde las primeras páginas que la obra no debe prevalecer sobre la vida, también reconoce que «el atentado iba a hacerle vivir cada minuto como si fuera la última línea» ${ }^{20}$. Esta relación de la obra con la vida se irá ampliando a lo largo de las páginas a una reflexión sobre el sentido de esta escritura y sobre sus destinatarios, sin saber exactamente si estaba escribiendo para los vivos - para anunciarles que pronto volvería entre ellos-, o más bien para sus compañeros muertos en el atentado. El ejercicio de la escritura se convierte entonces en una interpretación musical: «Lección de piano póstumo: si la mano derecha interpreta para los vivos, la izquierda interpreta para los muertos y es ella la que bate la medida» ${ }^{21}$. Esta relación con la música será corroborada por la crítica:

En papel, [El colgajo] es un testimonio, un documento. Y, sin embargo, no es así. El colgajo no tiene el estilo (es una música discreta, la del alma, que acompaña al lector de punta a punta) ni la intención demostrativa. Quien busque los escalofríos del pathos, el fuerte alcohol

19 Raspiengeas, 2019.

20 «Siempre me molestan los escritores que dicen que escriben cada frase como si fuera la última de sus vidas. Es darle demasiada importancia a la obra, o demasiado poco a la vida. Lo que no sabía era que el atentado iba a hacerme vivir cada minuto como si fuera la última línea: olvidar lo menos posible se vuelve esencial cuando te vuelves brutalmente ajeno a lo que has vivido [...]. Lançon, op. cit., p. 27.

21 lbíd., p. 205. 
de la política, el estruendo del choque de civilizaciones, puede pasar de largo, esta lección de tinieblas ${ }^{22}$, como el lamento de la viola da gamba, cuelga de un hilo mucho más tenue: el de la existencia ${ }^{23}$.

Una de las consecuencias de esta experiencia tan particular de la literatura es que aparece como una primera etapa hacia otras artes: tanto es así que la música actúa como una anestesia que trasciende el ámbito físico para curar también el alma: la música de Bach se vuelve una morfina que «eliminaba toda tentación de denuncia, cualquier sentimiento de injusticia, cualquier extrañeza del cuerpo» ${ }^{24}$.

La exposición de las obras de Velázquez a la que puede asistir durante su convalecencia es un acto vital que lo devuelve a sí mismo, cuando la contemplación de «las miradas intensas y marginales» ${ }^{25}$ de los bufones del pintor español con sus discapacidades se vuelve un espejo tranquilizador de su propia situación. Es el momento en que la pintura «prevalece sobre la literatura en el impulso físico hacia la vida ${ }^{26}$. La escritura se vuelve entonces un acto paradójico, un juego laberíntico junto con la pintura:

Nunca había experimentado tanto la sentencia profética: la escritura sí era el producto de otro yo, un producto precisamente destinado a sacarme del estado en que me encontraba, aunque consistía en contar este estado. Escribía sobre un cuadro de Velázquez en Libération como escribía sobre mi historial quirúrgico en Charlie, para entrar en el primero y escapar del segundo ${ }^{27}$.

También el cine participa de esta toma de conciencia del paciente, en particular cuando, en una entrevista con el periódico La Croix, Philippe Lançon recuerda que uno de los personajes de la película de Stefan SpieIberg En busca del arca perdida (1981) debe poner un pie en el vacío para que se edifique un puente encima del precipicio: escribir le parece entonces semejante a esta acción de poner un pie en el vacío, edificando un puente hacia la otra orilla de los lectores» ${ }^{28}$.

22 Las Lecciones de tinieblas (1714), de François Couperin (1668-1733), son ciclos de tres piezas basados en el Libro de las lamentaciones de Jeremías, que se interpretaban en Francia cada Jueves, Viernes y Sábado Santos hacia las tres de la mañana, entre tinieblas.

23 Trémolet de Villers, 2018.

24 «La música de Bach, como la morfina, me aliviaba. Hacía algo más que aliviarme: eliminaba toda tentación de denuncia, cualquier sentimiento de injusticia, cualquier extrañeza del cuerpo. Bach descendía sobre la habitación y la cama, y mi vida, sobre las enfermeras y su carro». Lançon, op. cit., p. 265.

25 lbíd., pp. 424-425.

26 lbíd., p. 427.

27 Ibíd., p.444

28 Raspiengeas, op. cit. 


\section{Trauma testimonio y creación, o la fuerza de la vulnerabilidad}

Entre las múltiples reflexiones que suscitan la experiencia y el texto de Philippe Lançon se plantea la cuestión de la literatura en su relación con el duelo y el testimonio de la víctima.

En un ensayo publicado en 2015, Los libros cuidan de nosotros, Régine Detambel dedica dos capítulos a «la poética del pathos» ${ }^{29}$ analizando la cuestión del duelo. Cita al novelista francés Philippe Forest, que tras la muerte de su hija fallecida de cáncer a los tres años, confió que lo había salvado la obra de Kenzaburō Ōe, cuyo hijo autista había nacido con profundas discapacidades ${ }^{30}$. Para sobreponerse a este dolor, el escritor japonés había decidido someterse a un dolor todavía mayor visitando Hiroshima en 1963, año del nacimiento de su hijo. Impactado por un dolor semejante, Philippe Forest escribirá El niño eterno dedicado a su hija ${ }^{31}$. Piensa, sin embargo, que si la novela puede encargarse de la experiencia humana de cada ser humano, no debe «curar del dolor de vivir». Para él, la literatura auténtica no debe consolar con virtudes terapéuticas; todo lo contrario, la novela verdadera no viene a ordenar el caos, sino «a despertar escandalosamente un desastre que se convierte a su vez en experiencia de verdad y de belleza» ${ }^{32}$. Como en eco a esta observación de Philippe Forest, Régine Detambel recuerda esta frase de Kafka a su amigo Oskar Pollak, que podría aplicarse a las dos narraciones y a la que hubiera podido referirse Philippe Lançon: «necesitamos libros que actúen sobre nosotros [...] Un libro debe ser el hacha para el mar helado que llevamos dentro» ${ }^{33}$.

Más allá del valor que presenta el testimonio de la víctima, el texto de Philippe Lançon nos incita también a preguntarnos sobre la respuesta de las artes y de las humanidades a la cuestión de la vulnerabilidad. En

29 Detambel, 2015.

30 «Hikari Ōe es un compositor japonés que padece autismo. Es hijo del escritor y ganador del Premio Nobel Kenzaburō Ōe. Nació con unas discapacidades que lo condenaban a la muerte según los médicos, pero sus padres rechazaron esta perspectiva. Tras una operación, permaneció con discapacidad visual, retraso en el desarrollo, epilepsia y una coordinación física limitada. Tampoco podía hablar mucho. Se dice que yendo de paseo con sus padres Hikari oyó el cantar de un pájaro y lo imitó con gran precisión. Fascinados, sus padres le compraron entonces grabaciones de audio con cantares de pájaros y contrataron a una profesora de piano, Kumiko Tamura, para enseñarle música. Hikari comenzó a expresar sus sentimientos a través de la música y mediante composiciones musicales, [hasta convertirse en] uno de los pianistas más virtuosos en la actualidad. Kenzaburō Ōe destacó la importancia de su hijo en su carrera literaria, dándole «Voz» a través de la escritura». El Negocio de la Música, 2017.

31 Forest, 1997. Philippe Forest es profesor de Literatura en la Universidad de Nantes. Ha escrito varios ensayos sobre la literatura y la historia de las corrientes vanguardistas y publicado siete novelas. Ha publicado una biografía de Louis Aragon, Aragon (París, Gallimard, 2015).

32 Detambel, op. cit., p. 36.

33 lbíd., p. 58. 
un reciente ensayo titulado El cuidado es un humanismo (2019) -con referencia a la obra de Jean-Paul Sartre El existencialismo es un humanismo (1946) - la filósofa y psicoanalista Cynthia Fleury desarrolla una visión humanista de la vulnerabilidad, que la lleva a una reflexión sobre el hospital como institución y sobre sus prácticas. En 2016, fundó la cátedra de Filosofía en el Hospital Hôtel-Dieu de París, y en la actualidad dirige la cátedra de Filosofía del hospital Santa Ana de París ${ }^{34}$, porque piensa que las humanidades deben tener un papel central, basado en la atención creadora para cada uno. En su ensayo desarrolla la idea según la cual la vulnerabilidad es una verdad de la condición humana que todos compartimos, aunque no estemos enfermos. Pero si la vulnerabilidad debilita al sujeto, presenta también la oportunidad de una posible sublimación, que es efectiva en la medida en que el individuo es capaz de reconquistar su «individuación» al enfrentarse con las diferentes pruebas existenciales que atraviesa, como lo muestran las vidas de determinados creadores. No se trataría, por consiguiente, de defender una concepción «dolorista» de la verdad en cuanto la experiencia del dolor sería una fuente para mejorar a la humanidad, sino de comprender que la vulnerabilidad puede ser capaz de trascender su propia condición ${ }^{35}$. El objetivo sería entonces acompañar al enfermo en «su reinvención de las normas de vida»; dicho de otro modo, de ayudarle a entrar en una dinámica de creación ${ }^{36}$.

\section{Conclusión}

Al referirse a la frase de Nietzsche «la concepción de la belleza para los griegos nació del dolor», Albert Camus observaba que para ellos el apolinismo y el dionisismo resultaban de la necesidad de huir de una vida demasiado dolorosa, caracterizada por las luchas políticas y todo tipo de violencias. De ahí la necesidad de crear formas o más bien sueños más bellos que en cualquier otro pueblo, sirviéndose de la danza y de la música para disciplinar su ebriedad mística y creando la tragedia ${ }^{37}$. El texto de Philippe Lançon, al contar una experiencia humana que, con un tono constantemente moderado y directo, relata una experiencia violenta, nos cuestiona sobre la función de la escritura y de la literatura frente a una situación traumática. Si en un determinado momento la lectura de

34 Cynthia Fleury (1974) es una filósofa y psicoanalista francesa, profesora asociada en la Escuela Normal Superior de Minas de París. Dirige la cátedra de Filosofía del hospital Saint Anne, Grupo Hospitalario Universitario de París, Psiquiatría y Neurociencias. Publicó varias obras, como Dialoguer avec l'Orient (Dialogar con Oriente) (PUF, 2003) o Les pathologies de la démocratie (Las patologías de las democracias) (Fayard, 2005).

35 Fleury, 2019, pp. 22-23.

36 Fleury, op. cit. p. 13.

37 Camus, 2006, p. 530. 
Saint-Simon logra disipar sus temblores físicos ${ }^{38}$, el narrador de El colgajo sabe también que no podrá ser el mismo al salir de esta experiencia y que su hospitalización habrá modificado no solo su cara, sino también su vida, su manera de leer, de escribir y de respirar. Esta experiencia nos incita a considerar la literatura en su relación con la enfermedad y la herida, no tanto como una terapia, sino como creación que nos permite tener acceso a un mundo diferente: el de un narrador que se libera del estatuto de víctima entrando en una dinámica de creación nacida del dolor.

\section{Contribuciones}

Jean-Marie Lassus ha participado en la concepción del artículo, la recolección de datos, su redacción y aprobación de la versión final.

\section{Fuentes de financiamiento}

Autofinanciado.

\section{Conflictos de intereses}

Ninguno. 


\section{REFERENCIAS BIBLIOGRÁFICAS}

Anagrama (2019). El colgajo. Recuperado de https://www.anagrama-ed. es/libro/panorama-de-narrativas/el-colgajo/9788433980410/PN_1009

Birnbaum, J. (11 de abril de 2018). Le lambeau, de Philippe Lançon: après Charlie, le journal du deuil. Le Monde des Livres. Recuperado de https:// www.lemonde.fr/livres/article/2018/04/11/apres-charlie-le-journal-dudeuil_5283979_3260.html

Camus, A. (2006). Nietzsche et la musique. En Fuvres complètes, París: Gallimard.

Desmeules, C. (5 de mayo de 2018). Le lambeau: I'homme qui a vu l'ours. Le Devoir. Recuperado de https://www.ledevoir.com/lire/526902/le-lambeau-I-homme-qui-a-vu-l-ours

Detambel, R. (2015). Les livres prennent soin de nous. Arles: Editions Babel Actes Sud.

El Negocio de la Música (2017). Hikari Ōe. «El milagro de la música». Recuperado de http://www.elnegociodelamusica.com/hikari-oe-milagro-lamusica/

Forest, P. (1997). L'enfant éternel. París: Gallimard. Traducido al español en 2007 como El niño eterno. Buenos Aires: Editorial del Estante.

Fleury, C. (2019). Le soin est un humanisme. París: Gallimard.

Foucault, M. (2011). Un peligro que seduce. Madrid: Cuatro Ediciones.

Lançon, P. (2018). Le lambeau. París: Gallimard. Traducido al español en 2019 como El colgajo. Barcelona: Anagrama.

Raspiengeas, J. C. (28 de enero de 2019). Philippe Lançon: «La parole apporte le soulagement de mieux se comprendre». [Entrevista]. La Croix. Recuperado de https://www.la-croix.com/Culture/Philippe-Lancon-paroleapporte-soulagement-mieux-comprendre-2019-01-28-1200998535

Torres Garza, E. (2017). El lenguaje sin hegemonía de Michel Foucault. Reflexiones Marginales. Recuperado de http://reflexionesmarginales. com/3.0/el-lenguaje-sin-hegemonia-de-michel-foucault/

Trémolet de Villers, V. (2018). Le lambeau, la leçon de ténèbres de Philippe Lançon. Le Figaro. Recuperado de https://www.lefigaro.fr/vox/ culture/2018/05/29/31006-20180529ARTFIG00246--le-lambeau-la-leconde-tenebres-de-philippe-lancon.php 